\title{
VERIFICATION OF PEOPLE BASED ON PALM VEIN WITH THE ROI AUTOMATIC APPOINTMENT
}

\author{
Dorota Smorawa \\ Institute of Computer and Information Sciences, Czestochowa University of Technology \\ Częstochowa, Poland \\ dorota.smorawa@icis.pcz.pl
}

\begin{abstract}
In the paper, the new approach to identification and verification of people based on the palm vein pattern will be presented. To create the feature vector, palm vein shape geometric arrangement will be applied. For detailed features will be considered similarly to minutiae which was applied in finger lines analysis in vein endings and bifurcation. The methods of vein location on the inner hand, methods of minutiae location, and features coding will be discussed in detail. To extract specific features a two-dimension density function will be used. The method to determine minutiae-vein bifurcation and endings will be presented.
\end{abstract}

Keywords: biometric system, recognition, palm vein

\section{Introduction}

One of the natural abilities of every human is the possibility to verify another person. Currently, a human lives in such times when he or she is required to log on to protected resources. We need an ID and a password, which can be easily lost or forgotten, to operate your bank account, social network or e-mail account. Every year many firms publish a list of the most often used IDs and passwords. Being trivial, they can be quickly broken with modern technology. Biometrics appears to be a helpful field in the unfair fight of the humans against the cyber world. This field of science deals with automatic recognition of people based on physical and behavioural features. Currently, there is variety of types of biometric systems based on signature [1], gait [2], fingerprint [3], hand geometry [4] or palm print [5, 6]. Each of these systems is to a more or less extent burdened with some defects. Some biometric features can be easily prepared, for example fingerprints. The next example can be a palm print when a finger, having been wounded, makes the print look different and will not be accepted by the system. The biometric system based on a palm vein does not have these defects. The vein pattern [7-9] is unique and unchanging for each person. This trait can be collected in a non invasive and a safe 
way. The blood vessels pattern cannot be faked because the vessels are under the skin. The acquisition of the pattern is carried out by lighting the hand with the near- infrared light. The image is taken with a CCTV active-matrix infrared camera. Furthermore, living features are automatically checked with the systems.

\section{Related work}

Presently, in the field of hand blood vessels research, a few interesting methods to create the biometric systems based on vein pattern can be found. In the papers [10], there is a description of the construction of a biometric system based on the palm vein, which uses a two-dimensional Gaussian function to create filters with which the image is being searched to find as many blood vessels as possible. The Hamming distance was used for pattern matching. In other works [11], a three-stage algorithm was proposed allowing to create the biometric system to identify people by means of blood vessels in a hand. The contrast enhancement and thresholding of hand blood vessels belong to the first stage of the project. The feature extraction and matching are based on the Gabor wavelet. In another research work [12], Jacobi algorithm is used to find vein and characteristic points. The well-known Euclidean distance was used for pattern matching.

A very essential matter is that the biometric system based on hand features, such as blood vessels or hand lines, has a method which determines the region of interest (ROI), which will be used when being constructed. This method allows for not applying any auxiliary plate which requires the hand to be positioned in a special way. The application of ROI lets the user position the hand during the acquisition in not a standard way, which significantly enhances the comfort of using such a device.

Presently, there are many methods to determine the region of a hand (ROI). All of these methods have the same assumption in common to always consider the same hand's region where the hand rotates during acquisition. In the work [13], the authors describe the ROI method which is used in biometric system research based on a palm print. In the first steps the image is binarized, next the methods to localize the edges are used. On such an image, the points between fingers are identified through which the parallel line to the image edge is placed. The next step in the method is drawing two straight lines at a $50^{\circ}$ angle to the earlier marked parallel line from the marked minimum points between the fingers. Next, the vertices of the ROI are determined. On such a selected image, extraction of detail features is applied. In this case it means localization of the maximum number of palm lines with the Discrete Cosine Transform. To compare the patterns saved in the base, the Hamming distance is employed. In another work [14], which describes how to recognize people based on their hand lines, the authors offer a very interesting method to determine ROI. Image binarization and the hand edge selection constitute the first steps, as it happened in the previous method. The next step in the method 
is to determine local minimum points between the fingers. The authors consider the left hand in these methods. The two valleys beside the middle finger are connected to form a reference line on the left side. Next, the reference line is extended to intersect the right-edge of the hand. The intersection point obtained from the previous step two is used to find the midpoint. The second two midpoints are connected to form the base line to obtain the ROI. Based on the principal of geometrical square, where all four edges have equal length, the other two points, needed to form the square outline of the ROI can be obtained. In another work [15] a very interesting touch-less palm print recognition system was proposed. In this approach the hand tracking and the ROI extraction step consists of three stages. In the first step, the hand image is segmented from the background using the skincolour thresholding method. After that, a valley detection algorithm is used to find four valleys of the fingers. Then a line is formed between the index finger valley and the little finger valley. Next, a square is drawn below the line. To feature extraction, the Local Binary Pattern is applied. The feature matching is based on the Chi-square measure. In other work [16] the author proposed a four-step algorithm for palm vein recognition. The first step is paramount, because it shows how the acquisition of the palm vein midpoint is searched for in the same way with the use of further two local minimums, images operate as a non-contact system. After the acquisition, the palm region is segmented by Otsu's method. Next, the palm border is extracted by the inner border tracing algorithm. After that, two data points, the index finger and the little finger are selected and through these points line $\mathrm{A}$ is constructed. Then the palm image is rotated between $\mathrm{A}$ and the horizontal line. In the end, a square region is located and denoted as the ROI, based on line A, with an angle. Next, the principal vein features are extracted by applying the modified (2D) $)^{2}$ LDA method. The modified $(2 \mathrm{D})^{2} \mathrm{LDA}$, which works in the column direction of images in the low dimensional 2DLDA subspace, can increase the recognition rate while reducing the coefficients of the feature matrix. The main advantage of modified (2D) $)^{2} \mathrm{LDA}$ over $(2 \mathrm{D})^{2} \mathrm{LDA}$ and $(2 \mathrm{D})^{2} \mathrm{PCA}$ is that fewer coefficients are needed for palm vein representation and recognition, saving computing time. Vein features are then matched with those from the template library by the minimum distance classifier (MDC) to verify the identity of the person.

\section{Palm vein image collection}

The biometric vein pattern is located under the skin. To activate the image of the hand vascular system, the infrared light and the active matrix infrared camera should be used. The near infrared light is partially absorbed by hemoglobin present in veins which creates a picture of the structure beneath the outer layer of the skin, presenting the natural contrast pattern of the blood vessels. The test stand consists of a CCTV active-matrix infrared camera and the IR lamp. 


\section{Proposed method for the ROI}

Defining the region of interest (ROI) is one of the most important steps in creation of the biometric system based on the hand blood vessels. Employment of the ROI allows for building a biometric system and facilitates the user's authorization. One of the first steps in the proposed method is extracting of the hand area by binarization. After that, the palm image is subjected to its own tracking method (Fig. 1), which is applied to estimate the palm region, as shown in Figure 2.

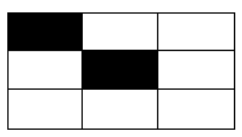

Condition 1

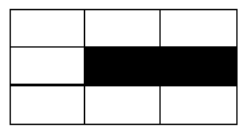

Condition 4

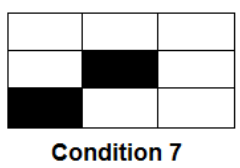

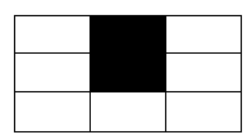

Condition 2

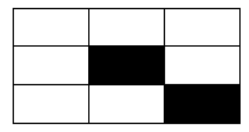

Condition 5

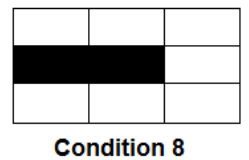

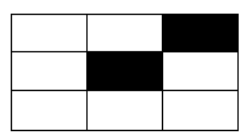

Condition 3

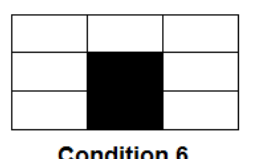

Condition 6

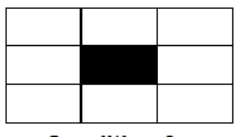

Condition 9

Fig. 1. Conditions of creating the tracking method

Next, the binarized palm shapes are compared with the original palm image in order to check the correct operation of the tracking method. Then, the points $P_{1}, P_{2}, P_{3}$ are determined. These points define the hand border between fingers. Through the $P_{1}$ and $P_{2}$ points the line $S$ is placed. The parallel line $L$ is appointed to the lower edge of the image which, at the same time, intersects the point $P_{1}$. The angle $\Theta(1)$ between the $S$ and $L$ lines is used to rotate the hand with respect to the coordinate system:

$$
\Theta=a \tan \left(\frac{Y P_{2}-Y P_{1}}{X P_{2}-X P_{1}}\right)
$$

The next step is connecting $P_{1}$ and $P_{2}$ points. The distance between those points defines the height and the width of the determined area. 


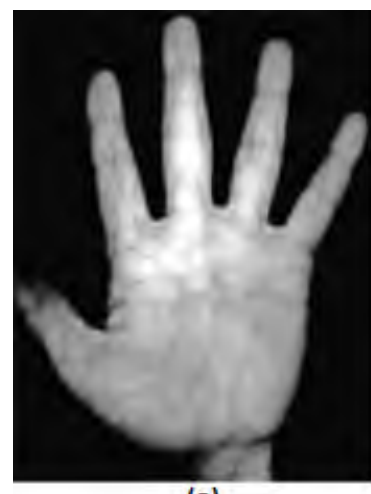

(a)

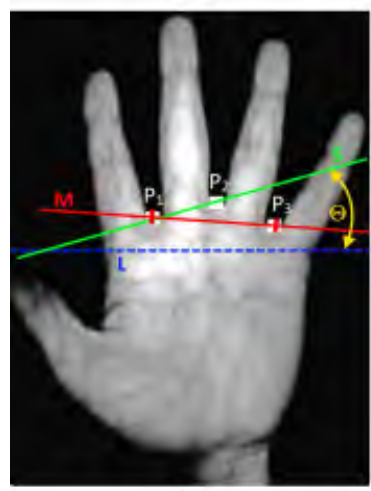

(c)

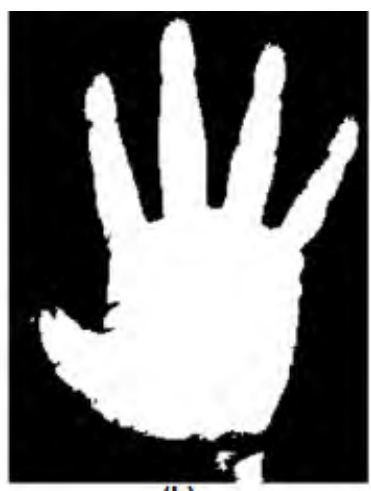

(b)

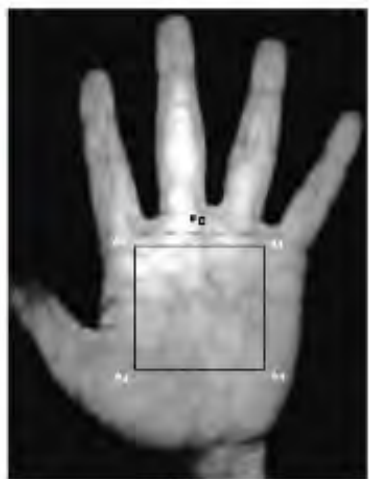

(d)

Fig. 2. Palm vein image preprocessing: a) original palm vein, b) binary palm vein image, c) two data points $P_{3}$ and $P_{2}$ are selected and the palm image is rotated by angle $\Theta$ between

lines $S$ and $L, \mathrm{~d}$ ) a square region is located and denoted as the ROI based on line M

\section{Extraction of palm vein based on two-dimensional density function}

The pattern of palm blood vessels in the image looks like a dent, because the veins are darker than the surrounding area. Our method examines the entire profile of the hand, pixel by pixel, and finds its value over a specified threshold, in order to capture the curvature of the image. This method is based on a two-dimensional density function (2) [17], presented below:

$$
f(x, y)=\frac{1}{2 \prod \delta^{2}} e^{\left(\frac{-\left(x^{2}+y^{2}\right)}{2 \delta^{2}}\right)}
$$

One of the first steps of my method is the initial location of curvature in the horizontal, vertical and both diagonal directions. For modelling, the curvature localizing filter the first (3), (4), (5) and the second (6), (7) derivatives of the two-dimensional density function are used. 


$$
\begin{gathered}
f_{x}=\frac{\partial f(x, y)}{\partial x}=\left(\frac{-x}{\delta^{2}}\right) f(x, y) \\
f_{x x}=\frac{\partial^{2} f(x, y)}{\partial x^{2}}=\frac{x^{2}-\delta^{2}}{\delta^{4}} f(x, y) \\
f_{y}=\frac{\partial f(x, y)}{\partial y}=\left(\frac{-y}{\delta^{2}}\right) f(x, y) \\
f_{y y}=\frac{\partial^{2} f(x, y)}{\partial y^{2}}=\frac{y^{2}-\delta^{2}}{\delta^{4}} f(x, y) \\
f_{x y}=\frac{\partial^{2} f(x, y)}{\partial x \partial y}=\frac{x y}{\delta^{4}} f(x, y)
\end{gathered}
$$

The filters are designed to locate all the existing curvature of the profile for the four directions. Filters for the horizontal direction (8), vertical (9) and two diagonal ones (10), (11) are described by the following formulas:

$$
\begin{gathered}
C_{d 1}(z)=\frac{f_{x x} \cdot L}{\left(1+\left(f_{x} \cdot L\right)^{2}\right)^{\frac{3}{2}}} \\
C_{d 2}(z)=\frac{f_{y y} \cdot L}{\left(1+\left(f_{y} \cdot L\right)^{2}\right)^{\frac{3}{3}}} \\
C_{d 3}(z)=\frac{0.5 f_{x x} \cdot L+f_{x y} \cdot L+0.5 f_{y y} \cdot L}{\left(1+\left(0.5 \cdot \sqrt{2}\left(f_{x} \cdot L+f_{y} \cdot L\right)\right)^{2}\right)^{\frac{3}{2}}} \\
C_{d 4}(z)=\frac{0.5 f_{x x} \cdot L-f_{x y} \cdot L+0.5 f_{y y} \cdot L}{\left(1+\left(0.5 \cdot \sqrt{2}\left(f_{x} \cdot L-f_{y} \cdot L\right)\right)^{2}\right)^{\frac{3}{2}}}
\end{gathered}
$$

where $L$ denotes the input ROI.

The next step is to determine the local maximal points $C_{d}(z)$ along the cross-section profile of the input image for all 4 directions $d$, where $z$ is a position in a cross-section profile (by one pixel). These points indicate the central position of the veins. This operation can be defined as $z_{i}$, where $i=0,1, \ldots, N-1$, and $N$ is the number of local maximum points in the cross-sectional profile. Next, scores 
indicating the probability that the center positions are on veins are assigned to each center positions. Score $P_{d}\left(z_{i}\right)$ is defined as follows (12):

$$
P_{d}\left(z_{i}\right)=C_{d}\left(z_{i}\right) N(i)
$$

The variable $N(i)$ is the width of the curvature area. At the same time, the designated curvature maximum points are assigned to the plane $V(x, y)$.

The next step is to connect the designated vein centers. This operation is used for all pixels designated in an earlier step. This action can be represented by the following formulas:

$$
\begin{aligned}
S_{d 1} & =\min \{\max (V(x+(m-1), y), V(x+m, y)) \\
& +\max (V(x-(m-1), y), V(x-m, y))\} \\
S_{d 2} & =\min \{\max (V(y+(m-1), x), V(y+m, x)) \\
& +\max (V(y-(m-1), x), V(y-m, x))\} \\
S_{d 3}= & \min \{\max (V(y-(m-1), x-(m-1)), V(y-m, x-m)) \\
+ & \max (V(y+(m-1), x+(m-1)), V(y+m, x+m))\} \\
S_{d 4}= & \min \{\max (V(y+(m-1), x-(m-1)), V(y+m, x-m)) \\
+ & \max (V(y-(m-1), x+(m-1)), V(y-m, x+m))\}
\end{aligned}
$$

where $m$ defines the scope of the filter $(m=2)$.

With designated a vein line for all four directions considered, the final pattern of blood vessels is formed by means of the function.

$$
F=\max \left(S_{d 1}, S_{d 2}, S_{d 3}, S_{d 4}\right)
$$

The last step is to bring the previously established pattern of blood vessels to a binary function, by the used thresholding.

At this stage the resulting pattern of blood vessels has a lot of noise and redundant information for the feature encoding process. To eliminate unnecessary disruption and vein discontinuity, four methods to improve the visibility of blood vessels have been applied. The first method is the dilatation, where the blood vessels are more protruded, which in time could result in a loss of relevant information about the position of the veins.

Then the thinning operation is performed. This operation reduces the size of the blood vessels to one pixel, making it easier to locate the veins fork.

After the dilation and thinning operations have been performed, there are still some irregularities on the image, and to smooth them out, some operations are carried out which remove unnecessary forks and image noise. 


\section{Encoding of features and matching}

In research to create feature vector, as a characteristic elements are used the minutiae in this case vein bifurcation and endings. That approach is used commonly in fingerprint analysis. The procedure of minutiae extraction is divided into four steps:

- $3 \times 3$ pixels sub-image is considered

- The nearest neighbourhood of a given pixel is searched

- The number of block pixels is computed

- If the number of block pixels equals exactly 2 (including the pixel being analyzed) $=$ the beginning or the end of the line

- If the number of block pixels equals 4 and 5 (including pixel being analyzed)

$=$ bifurcation

- Making the minutiae

The result of these methods can be seen in Figure 3.

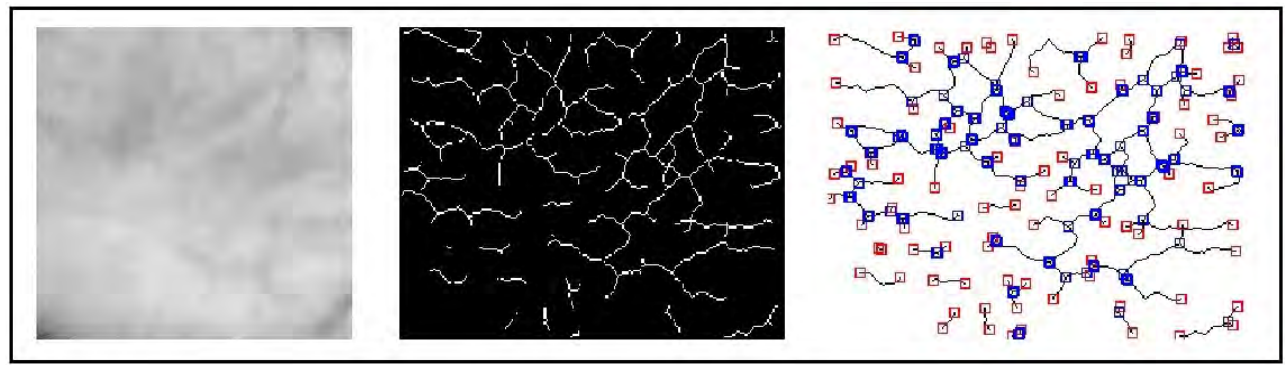

Fig. 3. The result of the detection method and minutiae extraction pf palm vein pattern

This method assumes that each pattern of blood vessels is described by the vector of features. Specific elements of this vector - minutiae - are described by coordinates of place, type and the position angle.

The problem of minutiae matching can by defined in the following way:

$$
\begin{aligned}
& T=\left\{m_{1}, m_{2}, \ldots, m_{m}\right\}, m_{i}=\left\{x_{i}, y_{i}, \theta_{i}\right\}, i=1 \ldots m \\
& I=\left\{m_{1}^{\prime}, m_{2}^{\prime}, \ldots, m_{n}^{\prime}\right\}, m_{j}^{\prime}=\left\{x_{j}^{\prime}, y_{j}^{\prime}, \theta_{j}^{\prime}\right\}, j=1 \ldots n
\end{aligned}
$$

where:

$T$ - vector of features saved in the data base,

$I$ - vector of features of blood vessels pattern being verified,

$m$ - the number of minutiae in the saved in the data base pattern,

$n$ - the number of minutiae in the pattern being verified.

Minutiae $m_{j}^{\prime}$ which belongs to $I$ is considered to be matching the minutiae $m_{i}$, which belongs to $I$, if their distance is shorter than the predetermined threshold $r^{0}$ and the difference of direction is smaller than the predetermined tolerance angle $\theta_{0}$ : 


$$
\begin{gathered}
s d\left(m_{j}^{\prime}, m_{i}\right)=\sqrt{\left(x_{j}^{\prime}-x_{i}\right)^{2}+\left(y_{j}^{\prime}-y_{i}\right)^{2} \leq r_{0}} \\
d d\left(m_{j}^{\prime}, m_{i}\right)=\min \left(\left|\theta_{j}^{\prime}-\theta_{i}\right|, 360^{\circ}-\left|\theta_{j}^{\prime}-\theta_{i}\right|\right) \leq \theta_{0}
\end{gathered}
$$

\section{Experiments results}

In this research work concerning the automated method for determining the ROI, mainly $P_{1}, P_{2}, P_{3}$ points and the correct identification of the users are presented. Experiments results were carried out based on the author's own data base. Each base contains data collected from 100 users with 12 pictures of the left and right hand each. For the teaching stage, 8 photos were used, and the remaining pictures were used in the tests. The results of the automated method for determining mainly points are shown in Table 1 .

Table 1

The results of the automated method

\begin{tabular}{|c|c|c|c|}
\hline $\begin{array}{c}\text { Point of the } \\
\text { number }\end{array}$ & $\begin{array}{c}\text { The number } \\
\text { of images hand }\end{array}$ & $\begin{array}{c}\text { Error along X } \\
\text { [pixel] }\end{array}$ & $\begin{array}{c}\text { Error along Y } \\
\text { [pixel] }\end{array}$ \\
\hline$P_{1}$ & 100 & 4.20 & 3.76 \\
\hline$P_{2}$ & 100 & 3.12 & 2.96 \\
\hline$P_{3}$ & 100 & 2.45 & 2.03 \\
\hline
\end{tabular}

Error along $X$ [pixel] - a average value of the error with respect to axis $\mathrm{x}$,

Error along $Y$ [pixel] - a average value of the error with respect to axis $\mathrm{y}$.

To check the level of security and accuracy of the systems, the factor of recognition rate was applied. The following table summarizes the results carried out for the different methods. The coefficients of proposed method shown in the Table 2 were obtained in tests which take into account the coding method based on the minutiae.

Table 2

The comparison of own method with other works

\begin{tabular}{|l|c|c|c|}
\hline \multicolumn{1}{|c|}{ Methods } & $\begin{array}{c}\text { Recognition rate } \\
{[\%]}\end{array}$ & $\begin{array}{c}\text { Feature vector } \\
\text { dimensions }\end{array}$ & $\begin{array}{c}\text { Matching time } \\
{[\mathrm{ms}]}\end{array}$ \\
\hline PCA [12] & 94.19 & 100 & - \\
\hline LDA [16] & 95.46 & 206 & - \\
\hline 2DPCA [16] & 98.07 & $64 \times 7$ & 2.23 \\
\hline 2DLDA [16] & 98.59 & $64 \times 6$ & 2.12 \\
\hline$(2 D)^{2}$ PCA [16] & 98.86 & $8 \times 8$ & 0.46 \\
\hline$(2 D)^{2}$ LDA [16] & $\mathbf{9 9 . 1 8}$ & $8 \times 8$ & $\mathbf{0 . 4 5}$ \\
\hline Minutiae feature [11] & 97.75 & - & - \\
\hline Laplacjanpalm [11] & 96.63 & $128 \times 128$ & - \\
\hline Eigenvein [11] & 98.31 & $128 \times 128$ & - \\
\hline Gaussian [11] & 98.88 & $64 \times 64$ & 324 \\
\hline Proposed & 99.08 & - & 15.04 \\
\hline
\end{tabular}




\section{Conclusion}

The author in this paper proposed the palm vein tracking algorithm to detect and locate the ROI of the palm vein automatically. A feature extraction method based on a two-dimensional density function was presented. The method to detect basic points, described in the work, which consists in tracking the outer edges of a hand is characterised by high accuracy, which explicitly enables the explicit determination of the ROI for each of the hand being analyzed. Such approach allows for the image acquisition when it is not necessary to position a hand in front of the scanning reader in an uncomfortable or schematic way. The result of the accuracy of basic points detection, presented in the work, indicate the easer point determination, which are local minimum between the fingers but only in the case when the fingers are not joined. The accuracy of detection is weakened in the other case. It is necessary to develop this method further, though.

The realization of searching for detailed features for correctly marked ROI, which are required for identification and verification of the users, proceeds smoothly, which was proved during the tests.

\section{References}

[1] Gluhchev G., Savov M., Boumbarov O., Vasileva D., A new approach to signature - based authentication, Advances in Biometrics, Lecture Notes in Computer Science 2007, 2, 594-603.

[2] Goffredo M., Spencer N., Pearce D., Carter J.N., Nixon M.S., Human perambulation as a self calibrating biometric, analysis and modeling of faces and gestures, Lecture Notes in Computer Science 2007, 1, 139-153.

[3] Kasban H., Fingerprints verification based on their spectrum, Neurocompiuting 2016, 171, 910-920.

[4] Sharma S., Dubey S.R., Singh S.K., Saxena R., Singh R.K., Identity verification using shape and geometry of human hands, Expert Systems with Applications 2015, 2, 821-832.

[5] Zheng P., Gaussian shape descriptor for palmprint authentication, Cogn. Comput. 2010, 2, 303-311.

[6] Kubanek M., Smorawa D., Adrjanowicz L., Users verification based on palm-prints and hand geometry with hidden markov models, Lecture Notes in Artificial Intelligence 2013, 7895, 1, $275-285$.

[7] Lee J.Ch., A Novel biometric system based on palm vein image, Pattern Recognition Letters 2012, 33, 1520-1528.

[8] Al-Juboori A., Bu W., Wu X., Zhao Q., Palm vein verification using multiple features and locality preserving projections, The Scientific World Journal 2014.

[9] Zhou Y., Liu Y., Feng Q., Yang F., Huang J., Nie Y., Palm-vein classification based on principal orientation features, PLOS ONE 2014, 9.

[10] Wu K-S., Lee J-C., Lo T-M., Chang K-C., Chang C-P., A secure palm vein recognition system, The Jurnal of Systems and Software 2013, 86, 2870-2876.

[11] Wang R., Wang G., Chen Z., Zeng Z., Wang Y., A Palm vein identification system based on gabor wavelet features, Neural Comput. \& Applic. 2014, 24, 161-168. 
[12] Sonal S-A., Dhiraj P., Pallavi D., Yogesh H-D., Hardware implementation of palm vein biometric modality for access control in multilayered security system, Procedia Computer Science 2015, 58, 492-498.

[13] Badrinath G.S., Gupta P., A Novel representation of palm-print for recognition, Lecture Notes in Computer Science 2011, 6493, 2, 321-333.

[14] Tee C., Teoh Beng Jin A., Goh Kah Ong M., Ngo Chek Ling D., An automated palmprint recognition system, Image an Vision Computing 2005, 23, 5, 501-515.

[15] Goh Kah Ong M., Tee C., Teoh Beng Jin A., Touch-less palm print biometric system, Image and Vision Computing 2008, 26, 1551-1560.

[16] Lee Y.P., Palm vein recognition based on a modifield (2D) ${ }^{2}$ LDA, Signal, Image and Video Processing 2015, 9, 1, 229-242.

[17] Smorawa D., Kubanek M., Biometric systms based on palm vein patterns, Journal of Telecommunications and Information Technology 2015, 2, 18-22. 